\title{
The Riemann Hypothesis and Pseudorandom Features of the Möbius Sequence
}

\author{
By I. J. Good* and R. F. Churchhouse
}

Abstract. A study of the cumulative sums of the Möbius function on the Atlas Computer of the Science Research Council has revealed certain statistical properties which lead the authors to make a number of conjectures. One of these is that any conjecture of the Mertens type, viz.

$$
|M(N)|=\left|\sum_{n=1}^{N} \mu(n)\right|<k(\sqrt{ } N)
$$

where $k$ is any positive constant, is false, and indeed the authors conjecture that

$$
\operatorname{Lim} \sup \left\{M(x)(x \log \log x)^{-1 / 2}\right\}=\sqrt{ }(12) / \pi \text {. }
$$

The Riemann zeta function is defined for $R(s)>1$ by the series $\zeta(s)=$ $\sum_{n=1}^{\infty} n^{-s}$, and the definition is completed by analytic continuation. Riemann's hypothesis is that the "complex zeros" all occur where $R(s)=\frac{1}{2}$. It has now been verified for the first 2,000,000 complex zeros (Rosser and Schoenfeld, [8]), but this is not a very good reason for believing that the hypothesis is true. For in the theory of the zeta function, and in the closely allied theory of the distribution of prime numbers, the iterated $\operatorname{logarithm} \log \log x$ is often involved in asymptotic formulae, and this function increases extremely slowly. The first zero off the line $R(s)=\frac{1}{2}$, if there is one, might have an imaginary part whose iterated logarithm is, say as large as 10, and, if so, it might never be practicable to find this zero by calculation. The plausibility of this argument is increased when we recall the refutation by Iittlewood [5] of the conjecture that $\pi(x)$, the number of primes less than $x$, is always less than the logarithmic integral, li $(x)$, a conjecture that is presumably true at least as far as $x=10^{9}$ (see Ingham [4, p. 7]). It is possible that Littlewood had this kind of argument in mind when he said (Littlewood [6]), "In the spirit of this anthology (an anthology of partly baked ideas) I should also record my feeling that there is no imaginable reason why it (the Riemann hypothesis) should be true."

The aim of the present note is to suggest a "reason" for believing Riemann's hypothesis.

The Möbius function is defined by $\mu(n)=(-)^{k}$ if the positive integer $n$ is the product of $k$ different primes, $\mu(1)=1$, and $\mu(n)=0$ if $n$ has any repeated factor. It is known (see, for example, Titchmarsh [9, p. 315]) that a necessary and sufficient condition for the truth of the Riemann hypothesis is that $M(x)=O\left(x^{1 / 2+\epsilon}\right)$, for all $\epsilon>0$, where $M(x)=\sum \mu(n)(n \leqq x)$. The condition $M(x)=O\left(x^{1 / 2+\epsilon}\right)$ would be true if the Möbius sequence $\{\mu(n)\}$ were a random sequence, taking the values $-1,0$, and 1 , with specified probabilities, those of -1 and 1 being equal.

Received May 1, 1967.

* Now at Virginia Polytechnic Institute, Blacksburg. 
More generally, if we first select a subsequence from $\{\mu(n)\}$ by striking out all the terms for which $\mu(n)=0$, and if this subsequence were 'equiprobably random,' i.e. if the value -1 and 1 each had (conditional) probability $\frac{1}{2}$, then the condition $M(x)=O\left(x^{1 / 2+\epsilon}\right)$ would still be true. Of course a deterministic sequence can at best be 'pseudorandom' in the usual incompletely defined sense in which the term is used, and of course all our probability arguments are put forward in a purely heuristic spirit without any claim that they are mathematical proofs.

As a matter of fact there are a priori reasons, that is without looking at the numerical support, for believing the following conjecture:

Conjecture A. The sums of $\mu(n)$ in blocks of length $N$, where $N$ is large, have asymptotically a normal distribution with mean zero and variance $6 \mathrm{~N} / \pi^{2}$.

A priori reasons for believing this conjecture. Note first that $\mu(n)=0$ when $n$ is a multiple of 4 , so there are zeros in the sequence $\{\mu(n)\}$ at regular intervals of 4 , and similarly at regular intervals of 9 and so on. So, if we write $\nu_{i}$ for the number of values of $n$ (in a block of length $N$ ) for which $\mu(n)=i$, we would expect $\nu_{0}$ to be very close to its expected value

$$
N-N\left(1-2^{-2}\right)\left(1-3^{-2}\right)\left(1-5^{-2}\right) \cdots=N\left(1-6 \pi^{-2}\right) .
$$

The numerical evidence for this statement is given in Tables 2 and 3 in the appendix.

Now if $n$ is large and known to be square-free it is likely to have a fair number of factors, and therefore by the theory of the roulette wheel (with two sectors instead of 37 or 38) the probabilities that the number of factors is odd or even are nearly equal. Thus the probability that $\mu(n)=1$ (or -1 ) is near to $3 \pi^{-2}$ and tends to this value when the range in which $n$ is known to lie tends to infinity. Hence the expectation of $\mu(n)$ is 0 .

The probability distribution of $\nu_{1}$, conditional on a knowledge of $\nu_{0}$, is binomial with mean $\frac{1}{2}\left(N-\nu_{0}\right)$ and variance $\frac{1}{4}\left(N-\nu_{0}\right)$. Allowing for the near-constancy of $\nu_{0}$, the unconditional distribution of $\nu_{1}$ would be expected to be binomial with mean $3 N \pi^{-2}$ and variance $3 N \pi^{-2} / 2$. As a matter of fact the variance does not depend on the near-constancy of $\nu_{0}$ since

$$
\begin{aligned}
\operatorname{var}\left(\nu_{1}-\nu_{-1}\right) & =E\left(\nu_{1}-\nu_{-1}\right)^{2} \\
& =E\left\{E\left[\left(\nu_{1}-\nu_{-1}\right)^{2} \mid \nu_{0}\right]\right\} \\
& =E\left(N-\nu_{0}\right)=6 N \pi^{-2}
\end{aligned}
$$

We have here assumed that, given $\nu_{0}, \nu_{1}$ has a 'heads-and-tails' binomial distribution. Its sample size is, of course, $N-\nu_{0}$.

This completes our a priori argument for believing Conjecture A, and even if Conjecture A is only approximately true it is so much stronger than the condition $M(x)=O\left(x^{1 / 2+\epsilon}\right)$ that we feel its approximate truth would still support that condition.

We now describe the numerical test of Conjecture A, which was performed with $N=1000$.

We computed $M(1000 r+1000)-M(1000 r$ ) (where we write $M(0)=0$ ) for $r=0(1) 49,999$ on the Chilton Atlas (as a "background" job) but the values $r=34,000(1) 34,999$ were lost owing to a machine fault. Column (ii) of Table 1 gives the frequencies with which 


$$
M(1000 r+1000)-M(1000 r)
$$

lies in the ranges given by column (i). The "expected values" of these frequencies, based on Conjecture A, are listed in column (iii). For example,

$$
7688=\frac{49}{\sigma \sqrt{ } 2 \pi} \int_{0.5}^{10.5} \exp \left(-\frac{1}{2} x^{2} / \sigma^{2}\right) d x,
$$

where $\sigma^{2}=6000 / \pi^{2}$. It will be seen that the fit is extremely good, in fact $\chi^{2}=24.2$ with 22 degrees of freedom. Thus Conjecture A is not merely to be expected $a$ priori, by mathematical common sense, but it is well supported by the numerical data. As we said before, we believe therefore that there is a good "reason" for believing the Riemann hypothesis, apart from the calculation of the first 2,000,000 zeros.

TABLE 1.

Frequencies of the values of $\sum \mu(n)(1000 r<n \leqq 1000(r+1))$, for 49000 values of $r$.

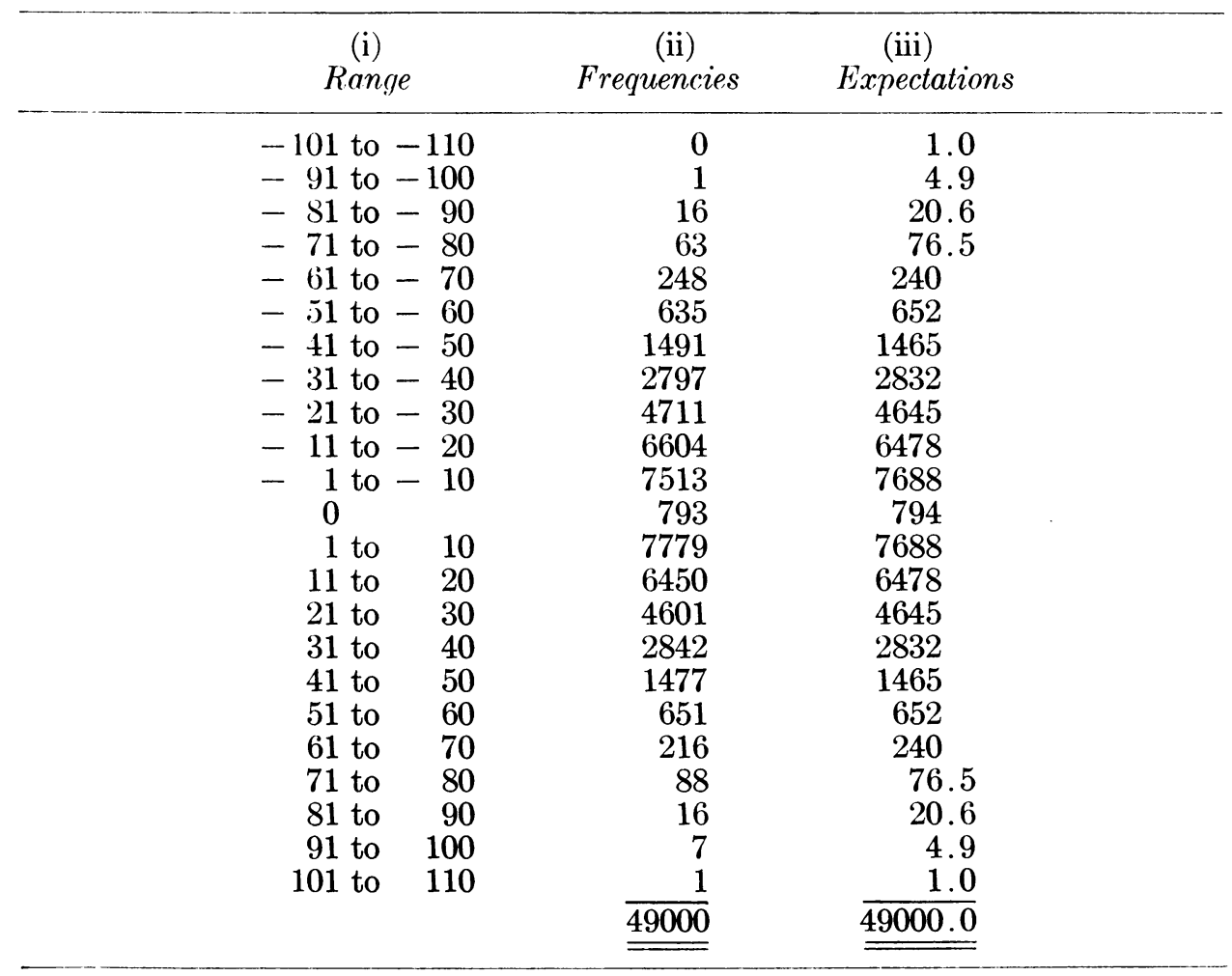

Although $\{\mu(n)\}$ is not a random sequence it is tempting to apply the law of the iterated logarithm (for example, Feller [1, p. 157]) to the subsequence obtained by deleting the values of $n$ for which $\mu(n)=0$. In this manner we generate a second conjecture, which, however, is less probable than Conjecture A and for which it is difficult to obtain numerical support. But it is of some interest to consider it. 
Conjecture B.

$$
\lim \sup \left\{M(x)(x \log \log x)^{-1 / 2}\right\}=\sqrt{ }(12) / \pi .
$$

Conjecture B contradicts Mertens's conjecture that $|M(x)|<x^{1 / 2}$, even in the extended form $|M(x)|<C x^{1 / 2}$ for any constant $C$. When $C=\frac{1}{2}$ this modification of Mertens's conjecture was refuted numerically by Neubauer [7]: a breakdown occurred, for example at $x=7.76 \times 10^{9}$. Also a conjecture of Pólya's, closely related to that of Mertens, was refuted by Haselgrove [3], who believed further that his method could be applied, with 1000 times as much calculation, to disprove Mertens's conjecture. In the light of this evidence, Mertens's conjecture is improbable, and our Conjecture B is somewhat supported by its inconsistency with it.

\section{Appendix. Distribution of $\nu_{0}$ for $N=1,000,000$}

In Table 2 we give the values of $\nu_{0}$, i.e. the number of cases of $\mu(n)=0$, in the first, second, . . ., 33rd block of length a million. We stopped at this point owing to the machine fault previously mentioned: a single supervisor fault caused the output for Table 1 to be lost at the 35th million and for Table 2 at the 34th million.

TABLE 2

\begin{tabular}{rccc}
\hline Million & $\nu_{0}$ & Million & $\nu_{0}$ \\
\hline 1 & 392,074 & 18 & 392,088 \\
2 & 392,049 & 19 & 392,039 \\
3 & 392,104 & 20 & 392,037 \\
4 & 392,037 & 21 & 392,072 \\
5 & 392,103 & 22 & 392,084 \\
6 & 392,076 & 23 & 392,096 \\
7 & 392,053 & 24 & 392,047 \\
8 & 392,101 & 25 & 392,096 \\
9 & 392,061 & 26 & 392,071 \\
10 & 392,051 & 27 & 392,071 \\
11 & 392,073 & 28 & 392,065 \\
12 & 392,078 & 29 & 392,079 \\
13 & 392,073 & 30 & 392,065 \\
14 & 392,095 & 31 & 392,083 \\
15 & 392,083 & 32 & 392,077 \\
16 & 392,093 & 33 & 392,076 \\
17 & 392,057 & & \\
\hline
\end{tabular}

Using the 33 values of $\nu_{0}$ given in Table 2, the estimated standard deviation is only 19.1. (The average number of zeros in each block of a million is very nearly 392,073 .) This suggests that nearly always $\nu_{0}$ is nearly constant in the sense that, for large $N$, the standard deviation of $\nu_{0}$ is $o(\sqrt{ } N)$, which for possible future reference we call Conjecture $\mathrm{C}$. The total expected number of zeros of the sequence $\{\mu(n)\}$ in the first $33,000,000$ is $33,000,000\left(1-6 \pi^{-2}\right)=12,938,405.6$ and the observed number is $12,938,407$, an astonishingly close fit, better than we deserved.

The values of $\nu_{0}$ for a few further values of $N$ are shown in Table 3.

On the basis of this table we might even strengthen Conjecture $\mathrm{C}$ to (Conjecture D) 'the variance of $\nu_{0}$ for large $N$ is a constant.' 
TABLE 3

\begin{tabular}{rrrl}
\hline$N$ & \multicolumn{1}{c}{$\nu_{0}$} & \multicolumn{1}{c}{$E\left(\nu_{0}\right)$} & $\nu-E\left(\nu_{0}\right)$ \\
\hline $25,000,000$ & $9,801,820$ & $9,801,822.5$ & -2.5 \\
$\tilde{5} 0,000,000$ & $19,603,656$ & $19,603,645$ & +11 \\
$75,000,000$ & $29,405,440$ & $29,405,467$ & -27 \\
$100,000,000$ & $39,207,306$ & $39,207,290$ & +16 \\
\hline
\end{tabular}

The conjecture of the near constancy of $\nu_{0}$ in each block of length $N$ must be interpreted in an average ('probabilistic') sense, whether or not it is expressed in the form of Conjecture C. It would not be correct to assume that $\nu_{0}$ is always close to its expected value; in fact it will sometimes though very rarely happen that $\nu_{0}=N$. This will happen, for example, in the block $(M+1, M+2, \cdots, M+N)$ if simultaneously $M \equiv-1(\bmod 4), \quad M \equiv-2(\bmod 9), \quad M \equiv-3(\bmod 25)$, $\cdots, M \equiv-N\left(\bmod p_{N}{ }^{2}\right)$, where $p_{N}$ is the $N$ th prime. These congruences can be solved by Sun-Tsu's theorem (see, for example, Good [2, p. 759]). The value of $M$ so obtained will be something like $N^{2 N}$. Thus the Möbius sequence $\{\mu(n)\}$ contains arbitrarily long runs of zeros, but these long runs presumably occur extremely rarely.

Atlas Computer Laboratory

Chilton, Didcot, Berkshire

England

Trinity College

Oxford, England

1. W. K. Feller, An Introduction to Probability Theory and its Applications, Vol. 1, Wiley, New York, 1950. MR 12, 424.

2. I. J. Good, "Random motion on a finite Abelian group," Proc. Cambridge Philos. Soc., v. 47,1951 , pp. $756-762$. MR 13, 363 .

3. C. B. HASELGRove, "A disproof of a conjecture of Pólya," Mathematika, v. 5, 1958, pp. 141-145. MR 21 \#3391. 1932.

4. A. E. Ingham, The Distribution of Prime Numbers, Cambridge Univ. Press, New York,

5. J. E. Littlewood, "Sur la distribution des nombres premiers," C. R. Acad. Sci. Paris, v. 158, 1914, pp. 1869-1872.

6. J. E. LiTTLEwood, "The Riemann hypothesis" in The Scientist Speculates, edited by Good, Mayne \& Maynard Smith, London and New York, 1962, pp. 390-391.

7. G. Neubauer, "Eine empirische Untersuchung zur Mertensschen Funktion," Numer. Math., v. 5, 1963, pp. 1-13. MR 27 \#5721.

8. J. B. Rosser \& L. Schoenfeld, "The first two million zeros of the Riemann zeta-function are on the critical line," Abstracts for the Conference of Mathematicians, Moscow, 1966, 8. (Unpublished.)

9. E. C. Titchmarsh, The Theory of the Riemann Zeta-Function, Clarendon Press, Oxford, 1951. MR 13, 741 . 